\title{
O Papel da Aspirina na Prevenção da Pré-Eclâmpsia: Estado da Arte
}

\author{
The Role of Aspirin in Preeclampsia Prevention: State of the Art
}

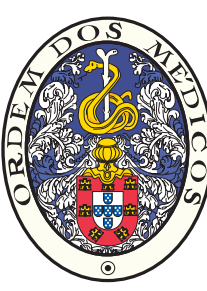

\author{
Ana CAMPOS $\square^{1,2}$ \\ Acta Med Port 2015 Jul-Aug;28(4):517-524
}

RESUMO

Introdução: O papel do ácido acetilsalicílico (AAS ou aspirina) na prevenção das complicações associadas à pré-eclâmpsia tem sido objeto de estudos e de controvérsias ao longo de 30 anos. Os primeiros trabalhos de investigação acerca do papel da placenta na génese da pré-eclâmpsia surgiram em finais dos anos 70 e assinalavam um aumento da atividade plaquetária e alteração da síntese das prostaglandinas, como consequência da deficiente adaptação da placenta. Ao longo dos últimos 20 anos do século XX, sucederam-se estudos de investigação acerca do papel profilático da aspirina na redução do risco de pré-eclâmpsia.

Material e Métodos: Para analisar os trabalhos publicados sobre o uso da aspirina na prevenção da pré-eclâmpsia, bem como sobre a dose mais adequada e momento de administração, foram consultados apenas estudos prospetivos, revisões sistemáticas e meta-análises através das seguintes fontes pesquisa (PubMed, Cochrane, Embase). Os artigos citados foram considerados os mais relevantes. Os trabalhos foram divididos em dois grupos: no primeiro foram incluídos os trabalhos em que a aspirina era administrada até às 16 semanas e o segundo, com início de administração por um período mais alargado.

Resultados e Discussão: No primeiro grupo, com menor número de casos, mas com início mais precoce de administração do fármaco, até às 16 semanas, concluiu-se que a aspirina poderia ter um papel positivo na redução de risco de gravidade da pré-eclâmpsia; o segundo grupo, com maior número de casos nos estudos, mas com condições menos restritas de entrada e de tempo de início do fármaco, teve resultados mais controversos. As meta-análises destes estudos concluíram que os resultados favoráveis estavam associados às condições de e momento da administração.

Conclusão: Não existindo ainda alternativas ou fármacos que lhe possam ser associados, a aspirina em baixas doses (80 a $150 \mathrm{mg} /$ dia) ao deitar, iniciada no $1^{\circ}$ trimestre e até às 16 semanas mantém-se um fármaco seguro, que tem contribuído para redução do risco de pré-eclâmpsia precoce, com as consequências que lhe estão associadas.

Palavras-chave: Aspirina; Pré-eclâmpsia/prevenção e controlo.

\section{ABSTRACT}

Introduction: The role of acetyl salicylic acid (ASA or aspirin) in preeclampsia prevention and in other complications has been subject to studies and controversies for the last 30 years. The first research results concerning the role of placenta in preeclampsia have been published by the end of seventies and they showed an increase in the platelet activity and a prostaglandin synthesis disturbance, as a consequence of a deficient placentation. In the last twenty years of the XX century important studies were published on the aspirin prophylactic role in preeclampsia risk reduction.

Material and Methods: To analyze published studies about Aspirin use for preeclampsia prevention and about the more adequate dosage to be administered, Medline was used for searching the most relevant prospective research papers on this subject in order to evaluate current evidence about the use of aspirin in this context. Relevant citations were extracted from Embase, PubMed and the Cochrane Central Register of Controlled Trials. We divided the studies in two groups; one with aspirine administration before 16 weeks and the other having a larger use, between the first and the third trimester.

Results and Discussion: The first group of studies, with a lesser number of cases but an earlier time of administration until 16 weeks, concluded that a positive role of aspirine was possible in reducing severity of preeclampsia; the second group with a larger number of cases but less restricted conditions and timing of administration, had controversial results, with reduced positive actions of the drug Meta-analysis of these published studies concluded that favorable results were associated to stricter criteria and ideal timing for starting the drug.

Conclusion: As we do not have other pharmacologic alternatives, low dosage of Aspirin between 80-150 mg a day in the first trimester and until 16 weeks, at evening time, is a possible choice in cases of risk, and is still contributing for an early preeclampsia risk reduction. Keywords: Aspirin; Pre-Eclampsia/prevention \& control.

\section{INTRODUÇÃO}

O ácido acetilsalicílico é usado como medicamento desde o antigo Egipto, há mais de 1000 anos. É retirado da casca do salgueiro e foi usado na medicina clínica no final do século XIX como analgésico e antipirético. Hoje a fórmula mais usada (aspirina) é o medicamento mais utilizado no mundo, mas só a partir dos anos 70 do século XX se percebeu o seu efeito na síntese das prostaglandinas. Inibidor

da agregação plaquetária que pode ocorrer em situações de lesão endotelial, estase vascular ou redução do fluxo sanguíneo, passou a ser usado na prevenção primária dos acidentes vasculares.

O papel dos salicilatos (AAS ou aspirina) na prevenção das complicações associadas à pré-eclâmpsia tem sido objeto de estudo e de controvérsias. Os primeiros trabalhos

\footnotetext{
1. Departamento de Obstetrícia. Maternidade Alfredo da Costa. Centro Hospitalar Lisboa Central. Lisboa. Portugal.

2. Faculdade de Ciências Médicas. Universidade Nova de Lisboa. Lisboa. Portugal.

$\triangle$ Autor correspondente: Ana Campos. anacampos.campos87@gmail.com

Recebido: 18 de Novembro de 2014 - Aceite: 22 de Junho de 2015 | Copyright @ Ordem dos Médicos 2015
} 
de investigação basearam-se na modificação da atividade e da agregação plaquetária nas mulheres com pré-eclâmpsia. ${ }^{1}$ Em finais dos anos 70 começou a ser considerado que uma deficiente adaptação placentária poderia ser um dos fatores explicativos da génese da pré-eclâmpsia, da modificação verificada nas plaquetas e na síntese de prostaglandinas, com um excesso de produção de tromboxano, um potente vasoconstritor. Masotti et $\mathrm{al}^{2}$ verificaram que a aspirina em baixas doses tinha um melhor efeito inibidor da ciclo-oxigenase nas plaquetas e nas paredes vasculares.

No processo fisiológico da placentação, a remodelação das arteríolas espiraladas inicia-se entre as 8 - 10 semanas, com a substituição da camada muscular da sua porção decidual pelas células do trofoblasto; numa segunda fase, entre as 14 e 16 semanas essa invasão atinge a porção intra-miometrial do vaso. ${ }^{2}$ Ao contrário do que sucede numa gravidez normal, nas mulheres destinadas a ter pré-eclâmpsia há desde cedo uma deficiente remodelação das artérias espiraladas, que abrange apenas a sua porção decidual. A consequência deste facto traduz-se na presença de vasos de alta resistência, com redução de fluxo vascular e hipóxia, libertação de substâncias pro-inflamatórias que lesam precocemente as células do endotélio, agregação plaquetária e alteração da síntese de prostaglandinas. ${ }^{3-5}$

Quimicamente as prostaglandinas são lípidos insaturados, biologicamente ativos, derivados do ácido araquidónico, constituídos por ácidos gordos com um anel ciclo-pentano; são sintetizadas em todas as células do organismo humano e têm múltiplas ações biológicas, atuando de forma semelhante a hormonas locais. Os glicerofosfolípidos, libertados pela ciclo-oxigenase, são a principal fonte de ácido araquidónico. Os dois principais compostos derivados do ácido araquidónico que atuam a nível do endotélio são a prostaciclina e o tromboxano. Têm ações antagónicas, tendo a prostaciclina (Pgl2) atividade endotelial essencialmente vasodilatadora e inibidora da atividade plaquetária e o tromboxano (TX) um potente vasoconstritor, uma atividade de promoção da agregação plaquetária (Fig. 1).

A pré-eclâmpsia, que ocorre em cerca de 2 a $8 \%$ das gravidezes, é uma doença multi-sistémica e tem diferentes graus de gravidade; pode ocorrer precocemente (antes das 34 semanas) havendo nesta situação maior gravidade no compromisso materno e fetal, ou ser de aparecimento tardio, habitualmente sem grande compromisso no crescimento fetal. Os critérios de classificação da doença têm vindo a ser modificados e atualmente, no critério de classificação de pré-eclâmpsia grave entram fatores maternos ou fetais (Tabela 1$){ }^{6}$

Não havendo ainda uma explicação completa para o aparecimento da doença, à teoria da má adaptação placentar estão associados outros fatores, nomeadamente genéticos e imunológicos que determinam fatores de risco para o seu aparecimento, com diferente importância relativa (Tabela 2).

Os fundamentos para o uso de aspirina, como anti-agregante plaquetário na redução da vasoconstrição,

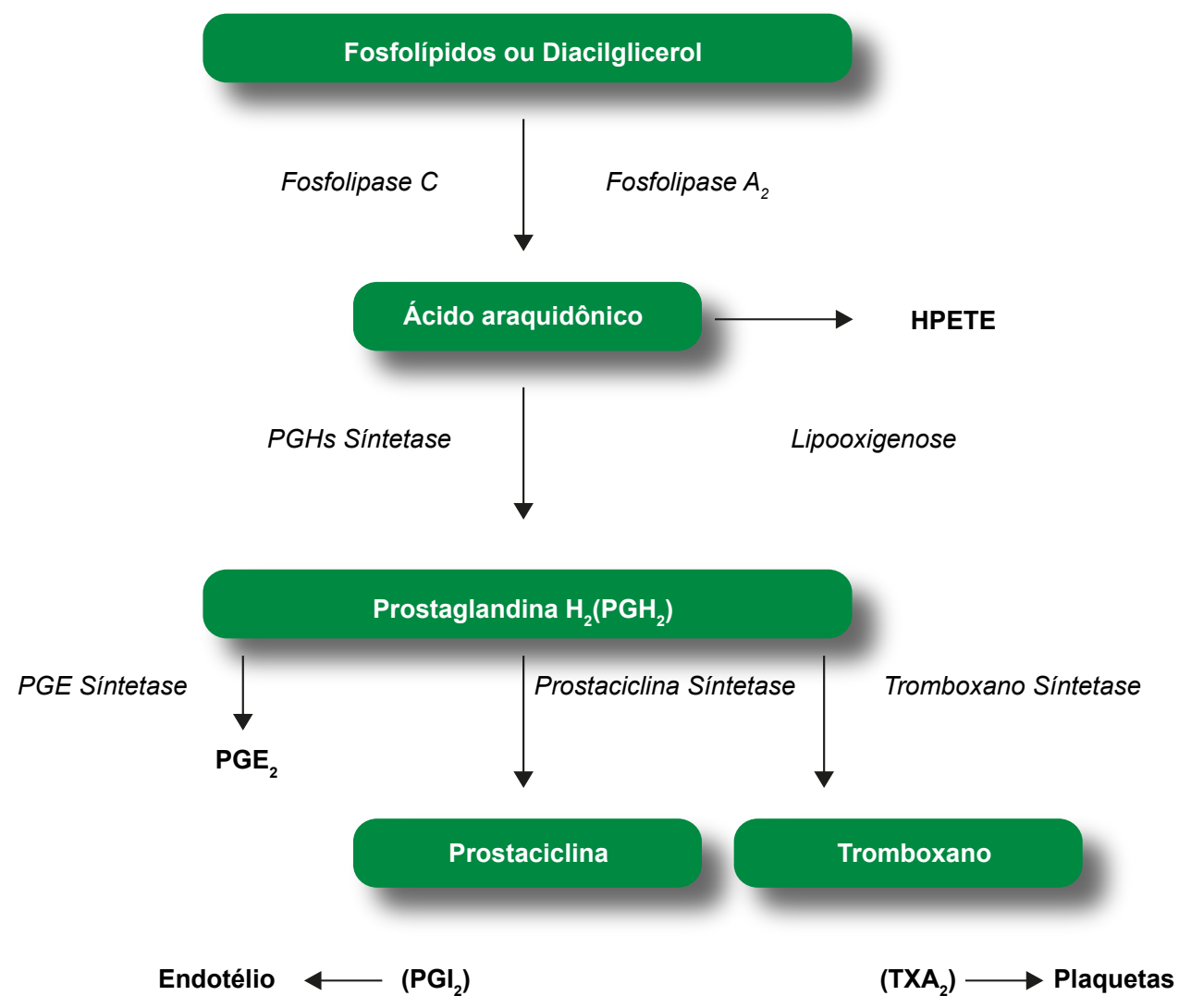

Figura 1 - Síntese das Prostaglandinas 
Tabela 1 - Critérios de classificação de pré-eclâmpsia

\begin{tabular}{|c|c|}
\hline Patologia & Definição* \\
\hline Hipertensão gestacional & $\mathrm{TA} \geq 140 / 90 \mathrm{~mm} \mathrm{Hg}$ em duas ocasiões com intervalo de 4 - $6 \mathrm{~h}$, iniciada após as 20 semanas \\
\hline Hipertensão crónica & $\mathrm{TA} \geq 140 / 90 \mathrm{~mm}$ existente previamente à gravidez, ou antes das 20 semanas \\
\hline Pré-eclâmpsia & $\begin{array}{l}\text { Hipertensão gestacional associada a proteinúria (+) em tiras teste ou } 100 \mathrm{mg} \text { em amostra } \\
\text { única (após as } 20 \text { semanas). }\end{array}$ \\
\hline Pré-eclâmpsia sobreposta & Hipertensão crónica associada a proteinúria \\
\hline Pré-eclâmpsia grave & $\begin{array}{l}\mathrm{TA} \geq 160 / 110 \mathrm{~mm} \mathrm{Hg} \text { e proteinúria } \geq 300 \mathrm{mg} / 24 \mathrm{~h} \text { ou } \\
\mathrm{TA} \geq 160 / 110 \mathrm{~mm} \mathrm{Hg} \text { e cefaleias, epigastralgias, restrição do crescimento fetal, edema } \\
\text { pulmonar, alteração das enzimas hepáticas }\end{array}$ \\
\hline Restrição do crescimento fetal & Peso ao nascer $<$ percentil 10 para a idade gestacional \\
\hline $\begin{array}{l}\text { Parto pré-termo } \\
\text { Parto pré-termo precoce }\end{array}$ & $\begin{array}{l}\text { Parto que ocorre antes das } 37 \text { semanas } \\
\text { Parto que ocorre antes das } 34 \text { semanas }\end{array}$ \\
\hline
\end{tabular}

${ }^{*}$ De acordo com as normas do Colégio Americano de Obstetrícia e Ginecologia (ACOG) $2013^{42}$

TA- Tensão arterial

baseiam-se no facto de, quando administrada em baixas doses, inibir o tromboxano $A_{2}$ (vasoconstritor) sem interferir com a produção de prostaciclina. ${ }^{2,5}$

Contudo, alguma controvérsia tem existido acerca do seu papel na profilaxia da pré-eclâmpsia. Os trabalhos publicados nas décadas de 80 e 90, dirigidos a parte ou várias situações de risco para esta patologia, apresentaram resultados controversos; diferem em relação ao número de casos estudados, mas também em relação aos limites de idade gestacional em que é iniciada a terapêutica com aspirina. As diferenças existem não só aos resultados de redução de risco de pré-eclâmpsia nas suas duas fases, mas também em relação ao papel da aspirina na redução do risco de restrição do crescimento fetal, como consequência de uma deficiente placentação.

O objetivo desta análise foi tentar obter evidências acerca do momento ideal para a terapêutica, destinatárias e dose adequada, bem como sobre os efeitos do fármaco em relação à redução de pré-eclâmpsia, nas suas formas precoce ou tardia, restrição do crescimento fetal e parto pré-termo.

\section{MATERIAL E MÉTODOS}

A pesquisa incidiu apenas em estudos prospetivos, com

Tabela 2 - Fatores de Risco para Pré-eclâmpsia

Gravidez anterior com pré-eclâmpsia
Hipertensão crónica
Doença renal
Diabetes mellitus tipo 1 ou tipo 2
Gestação múltipla
Doença auto-imune
Nuliparidade
História familiar de pré-eclâmpsia
Obesidade

randomização, meta-análises e revisões sistemáticas através das seguintes fontes (PubMed, Cochrane, Database). Com este objetivo, recolheram-se e analisaram-se os mais relevantes estudos obtidos entre 1979 e 2014, através das fontes de pesquisa citadas, tendo-se obtido a lista de estudos através do uso das palavras-chave e dos seguintes termos MeSH 'pregnancy', 'pregnancy-complication', 'aspirin', 'antiplatelet', 'preeclampsia', 'hypertension'. Escolheram-se apenas artigos publicados em língua inglesa ou francesa.

\section{RESULTADOS E DISCUSSÃO \\ Evolução e avaliação dos estudos}

Em 1979 Crandon e Isherwood ${ }^{7}$ constataram que as nulíparas que tomavam aspirina por situações variadas tinham menor incidência de pré-eclâmpsia. Em 1985 Beaufils et al ${ }^{8}$ publicaram o primeiro estudo randomizado com a utilização de $150 \mathrm{mg}$ de aspirina e $300 \mathrm{mg}$ de dipridamol, iniciados às 14 semanas de gravidez em mulheres com risco de pré-eclâmpsia, baseado na existência de hipertensão prévia ou de hipertensão em gestação anterior, demonstrando que esta terapêutica reduzia o risco de pré-eclâmpsia, restrição do crescimento e morte fetal neste grupo de alto risco. Seguiram-se publicações de 10 estudos randomizados, com administração de aspirina iniciada até às 16 semanas, entre 1985 e 2005 (Tabela 3). . $^{8-18}$

Estes trabalhos foram desenhados com utilização precoce de aspirina; nos grupos de estudo foi verificada redução de resultados desfavoráveis nos casos de risco de pré-eclâmpsia, o que acentuará a importância da sua utilização precoce, até às 16 semanas, momento em que deve estar terminada a segunda onda de invasão trofoblástica e completada a remodelação das arteríolas espiraladas. Contudo, estes trabalhos continham um número reduzido de casos e, embora metodologicamente corretos, os seus resultados necessitavam de ser replicados em estudos com maior número de casos.

Os estudos posteriores, entre 1993 e 2003, com maior 
Tabela 3 - Trabalhos escolhidos. Critério 1: Estudos prospetivos apresentados por ano, com início da administração de aspirina até às 16 semanas.

\begin{tabular}{|c|c|c|c|c|}
\hline Autor - Ano & $\begin{array}{l}\text { Participantes } \\
\text { n; IG início }\end{array}$ & Critérios de inclusão & Fármaco/dose & Resultado \\
\hline Beaufil $^{8} 1985$ & $102-14 s$ & $\begin{array}{l}\text { Patologia Grav. ant/HTA } \\
\text { prévia }\end{array}$ & $\begin{array}{l}\text { AAS } 150 \mathrm{mg}+\text { Dipiridamole } \\
300 \text { vs. s/tratamento }\end{array}$ & $\begin{array}{l}\text { IG parto;HIG/PE; } \\
\text { RCF; DPPNI }\end{array}$ \\
\hline Benigni $^{9} 1989$ & $33-12 s$ & HTC/Passado obst significativ & AAS 60 mg/vs. Placebo & HIG/PE/PPT/RCF \\
\hline Michael ${ }^{10} 1992$ & $110-16 s$ & HTA inicial/PE ant & AAS 100 mg/vs. Placebo & HG/PE \\
\hline August ${ }^{11} 1994$ & $54 ; 13-15 s$ & HTC/PE ant & AAS 100 mg/vs. Placebo & PE/RCF/DPPNI \\
\hline Hermida $^{12} 1997$ & $107 ; 12-16 s$ & $\begin{array}{l}\text { HIG;PEant;HTC; obesidade/ } \\
\text { nulipara/Gemelar/hist. fam de } \\
\text { HIG/PE }\end{array}$ & AAS 100 mg/vs. Placebo & $\begin{array}{l}\text { HIG/PE/RCF/PPT } \\
\text { IG parto/Peso RN/ } \\
\text { DPPNI }\end{array}$ \\
\hline Tuppala ${ }^{13} 1997$ & $66 ; 7 s$ & Aborto recorrente & ASA 50 mg/vs. Placebo & PE/RCF/peso RN \\
\hline Dasari'14 1998 & $50 ; 12 s$ & Nulípara & AAS 100 mg/vs. Placebo & $\begin{array}{l}\text { IGest.Parto/RCF/ peso } \\
\text { RN }\end{array}$ \\
\hline Hermida $^{15} 1999$ & $255 ; 12-16 s$ & $\begin{array}{l}\text { HIG;PEant;HTC; obesidade/ } \\
\text { nulipara/Gemelar/hist. fam de } \\
\text { HIG/PE }\end{array}$ & AAS 100 mg/vs. Placebo & RCF/PPT/DPPNI \\
\hline Vainio $^{16} 2002$ & $90 ; 12-14 s$ & $\begin{array}{l}\text { História pessoal e doppler } \\
\text { anormal das art. uterinas }\end{array}$ & Aas $0,5 \mathrm{mg} / \mathrm{Kb} / \mathrm{d} / v s$. Placebo & $\begin{array}{l}\text { HIG/PE/RCF/LIG/ } \\
\text { IG parto }\end{array}$ \\
\hline Chiaffarino $^{17} 2004$ & $40 ;<14 s$ & HTC/PE ant/ecl/RCF/FM & $\begin{array}{l}\text { AAS } 100 \mathrm{mg} / \mathrm{vs} . \mathrm{sem} \\
\text { tratamento }\end{array}$ & $\begin{array}{l}\text { HIG/RCF/Peso/IG } \\
\text { parto }\end{array}$ \\
\hline Ebrashy ${ }^{18} 2005$ & $139 ; 14-16 s$ & $\begin{array}{l}\text { Fluxometria doppler art } \\
\text { uterinas; fatores de risco para } \\
\text { PE e RCF }\end{array}$ & AAS $75 \mathrm{mg} / \mathrm{vs}$. sem tratamento & PE/RCF/PPT/Peso RN \\
\hline
\end{tabular}

Descolamento prematuro da placenta normalmente inserida; HTC: Hipertensão crónica; PPT: Parto pré-termo; RN: Recém-nascido

dimensão de participantes, igualmente prospetivos e randomizados, não concluíram na sua maioria pela vantagem da utilização de aspirina, quer na prevenção de pré-eclâmpsia, quer na redução de risco de restrição de crescimento fetal ${ }^{18-26}$ à exceção de dois trabalhos. Hauth ${ }^{19}$ e Sibai ${ }^{21}$ concluíram que existia uma redução de risco de pré-eclâmpsia em nulíparas saudáveis. Contudo Sibai, no grupo de estudo com aspirina encontra um aumento significativo de casos de abruptio placentae (Tabela 4).

Nestes estudos há aspetos que os diferenciam dos anteriores; a administração de aspirina não se limitou à sua utilização precoce. Encontram-se trabalhos em que a variabilidade de entrada no estudo é grande, entre as 12 e as 32 semanas. Observa-se também que havia grande disparidade de objetivos e indicações de tratamento, bem como populações com risco diferente e os seus resultados foram controversos.

Três trabalhos não incluídos neste grupo, para além dos objetivos principais já mencionados, procuraram avaliar a melhor dose de aspirina a utilizar. ${ }^{28-31} \mathrm{~A}$ avaliação da redução do risco de restrição de crescimento fetal com o uso de aspirina foi definida como objetivo principal num estudo multicêntrico, publicado em 1991, o estudo EPREDA. ${ }^{30}$ Concluiu que com a utilização entre as 15 - 18 semanas de aspirina na dose de $150 \mathrm{mg}$, associada a dipiridamol (225 $\mathrm{mg} / \mathrm{dia}$ ) houve prevenção do risco de restrição de cresci- mento fetal, em grávidas com este antecedente.

Vinikka et al, ${ }^{31}$ utilizando uma baixa dose $(50 \mathrm{mg})$ de ácido acetilsalicílico iniciado em média às 15 semanas em mulheres com hipertensão arterial prévia à gravidez ou pré-eclâmpsia grave em gestação anterior, verificou que, em comparação com os controles, os agentes anti-plaquetários não impediram a subida da pressão arterial materna, mas melhoraram os resultados fetais, com melhor resposta hemodinâmica fetal, redução de restrição de crescimento fetal e menor necessidade de internamento em unidades de cuidados intensivos neonatais. Verificaram uma forte inibição do tromboxano A2 materno, sem redução da produção de prostaciclina, preponderando portanto a dominância da ação vasodilatadora e de anti-agregação plaquetária.

Os estudos de maiores dimensões, multicêntricos, em especial o estudo CLASP 22 (Collaborative low-dose aspirin study in pregnancy), publicado em 1994, vieram refrear o otimismo existente. Com um número grande de casos (9 $364)$, este estudo tinha no entanto dois objetivos difíceis de conciliar: a administração de $60 \mathrm{mg}$ aspirina para objetivos profiláticos (hipertensão crónica, doença renal, antecedentes familiares de pré-eclâmpsia, idade materna e gestação múltipla) ou terapêuticos, cabendo ao clínico a iniciativa de introduzir o caso no estudo, se havia um quadro de pré-eclâmpsia ou de restrição de crescimento fetal já instalados. Podia assim ser iniciada a terapêutica com aspirina 
Tabela 4 - Trabalhos escolhidos. Critério 2: Estudos prospetivos apresentados por ano, com maior representação de casos mas sem critérios de início de uso de aspirina apenas até às 16 semanas.

\begin{tabular}{|c|c|c|c|c|}
\hline Autor - Ano & $\begin{array}{c}\text { Participantes } \\
\mathrm{n} \text {; Idade gestacional }\end{array}$ & Critérios de inclusão & Fármaco/dose & Resultado \\
\hline Hauth ${ }^{19} 1993$ & $606 ; 24 s$ & Nulíparas saudáveis & AAS 60 mg/vs. Placebo & HIG/PE/RCF/PPT/Peso RN \\
\hline Italian Study20 1993 & $1106 ; 16-32 s$ & $\begin{array}{l}\text { Idade }<18 \text { e }>40 A \text {; } \\
\text { HTC; Nefropatia; PE } \\
\text { ant e RCF ant }\end{array}$ & AAS $50 \mathrm{mg} / \mathrm{vs}$. Placebo & HIG;PE;Peso RN \\
\hline Sibai ${ }^{21} 1993$ & $2985 ; 13-26 s$ & Nulíparas saudáveis & AAS 60 mg/vs. Placebo & HIG/PE/RCF/Peso RN/DPPNI \\
\hline CLASP22 1994 & $9364 ; 12-32 s$ & $\begin{array}{l}\text { História pessoal de } \\
\text { Risco de PE; HTC; } \\
\text { Grav múltipla; } \\
\text { PE estabelecida; } \\
\text { RCF estabelecido }\end{array}$ & AAS 60 mg/vs. Placebo & PE/RCF/PPT \\
\hline ECPPA $^{23} 1996$ & $1009 ; 12-32 s$ & $\begin{array}{l}\text { HTC, nulípara, } \\
\text { diabetes, d. renal, PE } \\
\text { anterior ou D. Renal }\end{array}$ & AAS $60 \mathrm{mg} / \mathrm{vs}$. Placebo & $\begin{array}{l}\text { PPT, RCF, HIG, IG parto, } \\
\text { DPPNI }\end{array}$ \\
\hline Caritis $^{24} 1998$ & $2539 ; 13-26 s$ & $\begin{array}{l}\text { Diabetes prévia; HTC; } \\
\text { grav. Múltipla; PE ant }\end{array}$ & AAS 60 mg/dia/vs. Placebo & PE/RCF/PPT/Peso RN/DPPNI \\
\hline Rotchell ${ }^{25} 1998$ & $3697 ; 12-32 s$ & Todas as grávidas & AAS 75 mg/dia/vs. Placebo & $\begin{array}{l}\text { PE; HIG;RCF;PPT;Peso RN; } \\
\text { DPPNI }\end{array}$ \\
\hline Golding ${ }^{26} 1998$ & $6275 ; 12-32 s$ & Nulíparas & AAS $60 \mathrm{mg} / \mathrm{vs}$. Placebo & $\begin{array}{l}\text { HIG;PE;PPT;RCF;DPPNI; IG } \\
\text { parto }\end{array}$ \\
\hline Subtil $^{27} 2003$ & $3294 ; 14-20+6$ & Nulíparas & AAS 100 mg/vs. Placebo & PE \\
\hline
\end{tabular}

HTA: Hipertensão arterial; AAS: Ácido acetilsalicílico; IG: Idade gestacional; HIG: Hipertensão induzida pela gravidez; PE: Pré-eclâmpsia; RCF: Restrição do crescimento fetal; DPPNI: Descolamento prematuro da placenta normalmente inserida; HTC: Hipertensão crónica; PPT: Parto pré-termo; RN: Recém-nascido

entre as 12 e as 32 semanas, para critérios profiláticos ou com critérios de doença já instalada. O estudo concluiu que não se justificava o uso de agentes anti-plaquetários para fins profiláticos ou terapêuticos em grávidas com aumento de risco de pré-eclâmpsia ou restrição do crescimento fetal. Faz contudo a ressalva de o seu uso profilático poder ser justificado em mulheres com risco de pré-eclâmpsia grave e precoce, condicionando risco de grande prematuridade. De acordo com os resultados, poderia ser apropriado neste grupo de mulheres iniciar aspirina em baixas doses no início do segundo trimestre e foi confirmada a segurança do fármaco e a sua importância nas situações de alto risco para pré-eclâmpsia precoce.

Estes aspetos foram sublinhados por Uzan ${ }^{32}$ já que, para este autor e iniciador da justificação das bases fisiopatológicas para o uso de aspirina, terá havido uma excessiva expectativa sobre as possibilidades de atuação deste fármaco.

Em avaliações posteriores à publicação do estudo CLASP, Hermida et al $^{12}$ assinalaram a importância de encontrar a altura do dia em que a administração de aspirina traduz melhores efeitos. A ação anti-agregante plaquetária da aspirina é dependente da fase do ritmo circadiano em que é administrada, por duas ordens de razões: o estudo do efeito fármaco-cinético demonstrou que a clearance aumenta durante o dia em relação à noite e também que existe uma relação circadiana na produção de prostaciclina e tromboxano, com uma maior produção no período noturno. Daí que este autor tenha assinalado a importância da prescrição de anti-plaquetários à noite, por terem um efeito mais expressivo.

Durante um período longo, entre o resultado do estudo CLASP e 2007 não houve grande número de publicações sobre o tema.

Em 2007, Askie ${ }^{33}$ publicou uma meta-análise, o estudo PARIS (Perinatal Antiplatelet Review of International Studies) em que procurou avaliar as publicações que tinham por objetivo analisar o papel da aspirina em situações de alto risco para pré-eclâmpsia. Dos 36 trabalhos publicados entre 1985 e 2005 com os critérios previamente estabelecidos no seu estudo, ficou confirmado o papel da aspirina na redução do risco relativo de pré-eclâmpsia ou parto antes das 34 semanas em 10\% de casos, bem como de complicações graves associadas à pré-eclâmpsia.

Bujold ${ }^{34}$ numa meta-análise publicada em 2010 em que foram analisados estudos prospetivos randomizados publicados, envolvendo a administração de anti-plaquetários na dose de 50 a $150 \mathrm{mg} / \mathrm{dia}$, procurou respostas para as questões que se colocam hoje em debate sobre o papel da aspirina. Nos objetivos principais do estudo incluiu o aparecimento de pré-eclâmpsia; como objetivos secundários incluiu a incidência de pré-eclâmpsia precoce, restrição de crescimento fetal e complicações perinatais associadas a estas situações. Dos 34 estudos incluídos concluiu que a administração de aspirina antes das 16 semanas apresentava resultados com diferenças significativas em relação à ocorrência de pré-eclâmpsia (risco relativo [RR] 0,47, intervalo de confiança [IC] 95\% 0,34 - 0,65), 
prevalência de 9,3\% no grupo com tratamento comparados com $21,3 \%$ no grupo controle; parto pré-termo (RR 0,22, IC $95 \%$ 0,10 - 0,49, 3,5\% no grupo com tratamento, comparado com $16,9 \%$ no grupo controle), restrição do crescimento fetal (RR 0,44, IC 95\% 0,30 - 0,65, 7\% no grupo com tratamento, comparado com $16,3 \%$ no grupo controle) e pré-eclâmpsia grave (RR 0,09, 95\% IC 95\% 0,02-0,37, $0,7 \%$ no grupo com tratamento, comparado com $15 \%$ no grupo controle).

Em estudo posterior, ${ }^{35}$ o mesmo autor, avaliando a dose a utilizar, não encontrou diferenças significativas entre 80 mg ou $150 \mathrm{mg}$, podendo haver necessidade de ajustar a dose às necessidades de cada caso. O momento ideal de administração situar-se-á entre as 8 e as 16 semanas, baseado no processo fisiológico associado à placentação, no que diz respeito à invasão das arteríolas espiraladas que estará completa nesse momento da gestação. Sugere ainda que os melhores efeitos serão obtidos se a administração se iniciar entre as 8 - 10 semanas nas situações de alto risco para pré-eclâmpsia.

Depois destas meta-análises baseadas nos estudos anteriores, ressalta que a indicação para o uso de aspirina deve ser baseada em fatores de risco para o aparecimento ou repetição de uma pré-eclâmpsia ou de restrição do crescimento fetal (antecedentes de doença hipertensiva, vasculopatias, passado obstétrico associado a pré-eclâmpsia ou restrição do crescimento fetal e doenças auto-imunes). $O$ início da sua administração deve ser anterior às 16 semanas, havendo indicação de que a dose ideal se situa entre $80 \mathrm{mg}$ e $150 \mathrm{mg}$.

\section{Estado atual}

A fisiopatologia da pré-eclâmpsia surge precocemente na gravidez, estando ainda em estudo e por encontrar a totalidade das suas causas. Há outros fatores importantes na resposta vasoativa, para além do desequilíbrio na relação prostaciclina /tromboxano. ${ }^{36} \mathrm{~A}$ pesquisa desses outros fatores, essencialmente fatores angiogénicos, constituiu preocupação de muitos investigadores nos últimos 10 anos e serão poucos os estudos dedicados unicamente ao papel da aspirina, na última década. A atual investigação reside essencialmente em encontrar testes preditivos precoces, que identifiquem as situações de risco para pré-eclâmpsia precoce, uma vez que se trata das situações mais graves com maior morbi-mortalidade materna e perinatal associada, a quem se imponha fazer terapêutica com aspirina. ${ }^{37}$

A utilização no primeiro trimestre de uma combinação de fatores de risco maternos (obesidade, história pessoal e familiar de hipertensão, pré-eclâmpsia em gestação anterior ou restrição de crescimento fetal), associados ao valor da pressão arterial média (PAM) entre as 11 - 13 semanas e à fluxometria das artérias uterinas, constituíram os primeiros testes com combinação de indicadores de risco de pré-eclâmpsia. ${ }^{38}$ Seguiu-se a identificação de marcadores placentares como a proteína plasmática - a associada à gravidez (PAPP-A) que pode ter valores reduzidos em situações de risco de pré-eclâmpsia ou de restrição do crescimento fetal, mas que usada isoladamente, tem muitos falsos positivos. Este marcador associado ao fator de crescimento placentar (PIGF), uma proteína da angiogénese placentar que promove a vasodilatação e está reduzida no primeiro trimestre e durante a gravidez em mulheres com risco de pré-eclâmpsia de instalação precoce, melhora a sua preditividade. Em estudos conduzidos no Reino Unido a associação destes marcadores biofísicos e bioquímicos permitiu a possibilidade de realização de um algoritmo preditivo de risco de pré-eclâmpsia precoce ou de restrição do crescimento fetal. ${ }^{38-40}$ Leona Poon publicou em 2009 um estudo conduzido no Reino Unido, abrangendo 7797 gestações simples em que foram utilizados estes marcadores de risco de pré-eclâmpsia precoce, com identificação de $93,7 \%$ dos casos de pré-eclâmpsia precoce, $35,7 \%$ de pré-eclâmpsia tardia e $18,3 \%$ de hipertensão gestacional, com uma taxa de falsos positivos de $5 \%{ }^{38}$

Serão pois esses casos que mais poderão beneficiar da terapêutica profilática com aspirina iniciada até às 16 semanas. Num estudo elaborado por Block-Abraham et al, estes autores verificaram, contudo, que a administração de aspirina até às 16 semanas numa população com os fatores de risco incluídos nos estudos anteriores justificando a sua administração, não evitou o aparecimento de pré-eclâmpsia nas mulheres com PAM elevada no $1^{\circ}$ trimestre..$^{41}$

\section{CONCLUSÃO}

Mantendo-se os mesmos critérios para iniciar a terapêutica com aspirina, como profilaxia da pré-eclâmpsia precoce, justifica-se a administração do fármaco em situações de risco para a sua ocorrência, tais como o passado de pré-eclâmpsia, doença vascular prévia, restrição de crescimento fetal em gravidez anterior, gestação múltipla, síndrome de anticorpo anti-fosfolípido ou aborto recorrente, assim como valores do algoritmo de risco preditivo acima de $10 \%$. As doses não estão claramente definidas ainda, mas a maior parte dos estudos e normas de orientação indicam que deve ser administrada uma dose entre 80 a $150 \mathrm{mg}$ de aspirina, administrada ao deitar e iniciada até às 16 semanas. ${ }^{42-45}$ Embora as doses e o momento de início sejam ainda temas controversos, a sua administração precoce tem justificação perante a sua ação no processo fisiopatológico. Nas situações de síndrome de anticorpo anti-fosfolípidos (SAAF) ou aborto recorrente, o início da terapêutica deve coincidir com o diagnóstico da gravidez evolutiva in-útero.

Não existindo hoje um marcador isolado que nos defina o risco de pré-eclâmpsia, mantém-se a necessidade de desenvolvimento de novos marcadores preditivos sobretudo do risco de pré-eclâmpsia precoce, que é a que está associada a maior morbilidade. A aspirina continua a ser até ao momento o fármaco de que se pode dispor, que pode ser usado de forma segura para a prevenção das situações de maior gravidade, contribuindo para uma redução da morbi-mortalidade materna e perinatal. 


\section{CONFIDENCIALIDADE DOS DADOS}

Os autores declaram ter seguido os protocolos do seu centro de trabalho acerca da publicação dos dados de doentes.

\section{CONFLITOS DE INTERESSE}

Os autores declaram não ter qualquer conflito de interesse relativamente ao presente artigo.

\section{FONTES DE FINANCIAMENTO}

Este trabalho não recebeu qualquer contribuição e subsídio ou bolsa.

\section{REFERÊNCIAS}

1. Redman CW, Bonnard J, Beilin L. Early platelet consumption in preeclampsia. Br Med J. 1978;1:467-9.

2. Roberts JM, Redman CW. Pre-eclampsia: more than pregnancyinduced hypertension. Lancet. 1993;341:1447-52.

3. Masotti G, Galanti G, Poggesi L, Abbate R, Neri Serneri GG. Differential inhibition of prostacyclin production and platelet aggregation by aspirin. Lancet. 1979;2:1213-7.

4. Janes SL, Kyle PM, Redman C, Goodall AH. Flow cytometric detection of activated platelets in pregnant women prior to the development of pre-eclampsia. Thromb Haemost.1995;74:1059-63.

5. Thorp JA, Walsh SW, Brath PC. Low-dose aspirin inhibits thromboxane, but not prostacyclin, production by human placental arteries. Am J Obstet Gynecol. 1988;159:1381-4.

6. Steegers EA, Dadelszen P, Duvekot JJ, Pijnenborg R. Pre-eclampsia Lancet. 2010;376:631-44.

7. Crandon AJ, Isherwood DM. Effect of aspirin on incidence of preeclampsia. Lancet. 1979;1:1356.

8. Beaufils M, Uzan S, Donsimoni R, Colau JC. Prevention of preeclampsia by early antiplatelet therapy. Lancet. 1985;1:840-2.

9. Benigni A, Gregorini G, Frusca T, Chiabrando C, Ballerini S, Valcamonico A, et al. Effect of low-dose aspirin on fetal and maternal generation of thromboxane by platelets in women at risk for pregnancyinduced hypertension. N Engl J Med. 1989;321:357-62.

10. Michael CA, Walters BN. Low-dose aspirin in the prevention of preeclampsia: current evaluation. In: Teoh ES, Ratnam SS, Macnaughton $M C$, editors. Maternal physiology and pathology. The current status of gynaecology and obstetrics series. Carnforth: Parthenon Publishing Group Limited; 1992. p 183-9.

11. August P, Helseth G, Edersheim T, Hutson J, Druzin M. Sustained relase, low-dose aspirin ameliorates but does not prevent preeclampsia $(\mathrm{PE})$ in a high risk population. Proceedings of the 9th International Congress. Sydney: International Society for the Study of Hypertension; 1994. p. 72.

12. Hermida RC, Ayala DE, Iglesias M, Mojon A, Silva I, Ucieda R, et al Time-dependent effects of low-dose aspirin administration on blood pressure in pregnant women. Hypertension. 1997;30:589-95.

13. Tulppala M, Marttunen M, Soderstrom-Anttila V, Foudila T, Ailus K, Palosuo $\mathrm{T}$, et al. Low-dose aspirin in prevention of miscarriage in women with unexplained or autoimmune related recurrent miscarriage: effect on prostacyclin and thromboxane A2 production. Hum Reprod. 1997;12:1567-72.

14. Dasari R, Narang A, Vasishta K, Garewal G. Effect of maternal low dose aspirin on neonatal platelet function. Indian Pediatr. 1998;35:50711.

15. Hermida RC, Ayala DE, Fernandez JR, Mojon A, Alonso I, Silva I, et al. Administration time-dependent effects of aspirin in women at differing risk for preeclampsia. Hypertension. 1999;34:1016-23.

16. Vainio M, Kujansuu E, Iso-Mustajarvi M, Maenpaa J. Low dose acetylsalicylic acid in prevention of pregnancy-induced hypertension and intrauterine growth retardation in women with bilateral uterine artery notches. Br J Obstet Gynaecol. 2002;109:161-7.

17. Chiaffarino F, Parazzini F, Paladini D, Acaia B, Ossola W, Marozio L, et al. A small randomised trial of low-dose aspirin in women at high risk of pre-eclampsia. Eur J Obstet Gynecol Reprod Biol. 2004;112:142-4.

18. Ebrashy A, Ibrahim M, Marzook A, Yousef D. Usefulness of aspirin therapy in high-risk pregnant women with abnormal uterine artery doppler ultrasound at 14-16 weeks pregnancy: randomized controlled clinical trial. Croat Med J. 2005;46:826-31.

19. Hauth J, Goldenberg R, Parker R, Philips J, Cooper RL; DuBard $M$, et al. Low dose therapy to prevent preeclampsia. Am J Obstet Gynecol.1993;168:1083-93.

20. Low-dose aspirin in prevention and treatment of intrauterine growth retardation and pregnancy-induced hypertension. Italian study of aspirin in pregnancy. Lancet. 1993;341:396-400.
21. Sibai BM, Caritis, SN, Thom E, Klebanoff M, McNellis D, Rocco L, et al. Prevention of preeclampsia with low- dose aspirin in healthy, nulliparous pregnant women. N Engl J Med. 1993;329:1213-18.

22. CLASP: A randomised trial of low-dose aspirin for the prevention and treatment of pre-eclampsia among 9364 pregnant women. Clasp (collaborative low-dose aspirin study in pregnancy) collaborative group. Lancet. 1994;343:619-29.

23. ECPPA: randomised trial of low dose aspirin for the prevention of maternal and fetal complications in high risk pregnant women. ECPPA (Estudo Colaborativo para Prevencao da Pre-eclampsia com Aspirina) Collaborative Group. Br J Obstet Gynaecol. 1996;103:39-47.

24. Caritis S, Sibai B, Hauth J, Lindheimer MD, Klebanoff M, Thom E, et al. Low-dose aspirin to prevent preeclampsia in women at high risk. National Institute of Child Health and Human Development Network of Maternal-Fetal Medicine Units. N Engl J Med. 1998;338:701-5.

25. Rotchell YE, Cruickshank JK, Gay MP, Griffiths J, Stewart A, Farrell B, et al. Barbados Low Dose Aspirin Study in Pregnancy (BLASP): a randomised trial for the prevention of pre-eclampsia and its complications. Br J Obstet Gynaecol. 1998;105:286-92.

26. Golding J. A randomised trial of low dose aspirin for primiparae in pregnancy. The Jamaica Low Dose Aspirin Study Group. Br J Obstet Gynaecol. 1998;105:293-9.

27. Damien S, Goeusse P, Puech F, Lequien P, Biausque S, Breart G, et al. Essai Régional Aspirine Mère-Enfant (ERASME) Collaborative Group Aspirin $(100 \mathrm{mg}$ ) used for prevention of pre-eclampsia in nulliparous women: the Essai Régional Aspirine Mère-Enfant study (Part 1). Br J Obstet Gynaecol. 2003;110:475-84

28. Schiff E, Barkai G, Ben-Baruch G, Maschiach S. Low dose aspirin does not influence the clinical course of women with mild pregnancy-induced hypertension. Obstet Gynecol. 1990;76:742-4.

29. Barth W. Low dose aspirin for preeclampsia - the unresolved question. N Engl J Med. 1998;11:756-7.

30. Uzan S, Beaufils M, Breart G, Bazin B, Capitan C, Paris J. Prevention of fetal growth retardation with low-dose aspirin findings of the EPREDA trial. Lancet. 1991;337:1427-31.

31. Viinikka L, Hartikainen-Sorri AL, Lumme R, Hiilesmaa V, Ylikorkala O. Low dose aspirin in hypertensive pregnant women: effect on pregnancy outcome and prostacyclin-thromboxane balance in mother and newborn. Br J Obstet Gynaecol. 1993;100:809-15.

32. Uzan S, Merviel P, Dumont A, Berkane N, Beafils M, Bréart G. Aspirine: un retour aux sources plutôt qu'un big disappointment. Gynecol Obstet Fertil. 2000;28:359-62.

33. Askie LM, Duley L, Henderson-Smart DJ, Stewart LA. Antiplatelet agents for prevention of pre-eclampsia: a meta-analysis of individual patient data. Lancet. 2007;369:1791-98.

34. Bujold E, Roberge S, Lacasse Y, Bureau M, Audibert F, Marcoux S, et al. Prevention of preeclampsia and intrauterine growth restriction with aspirin started early in pregnancy: a meta-analysis. Obstet Gynecol. 2010;116:402-14

35. Bujold E, Roberge S, Demers S, Nicolaides KH. Aspirin administration for prevention of adverse pregnancy outcomes. Fetal Matern Med Rev. 2012;23:187-200.

36. Gilbert JS, Ryan MJ, LaMarca BB, Sedeek M, Murphy SR, Granger JP. Pathophysiology of hypertension during preeclampsia: linking placental ischemia with endothelial dysfunction. Am J Physiol Heart Circ Physiol. 2008:294;H541-50.

37. Roberge S, Nicolaides K, Demers S, Villa P, Bujold E. Prevention of perinatal death and adverse perinatal outcome using low-dose aspirin A meta-analysis. Ultrasound Obstet Gynecol. 2013;41:491-9.

38. Poon LC, Kametas NA, Maiz N, Akolekar R, Nicolaides KH. Firsttrimester prediction of hypertensive disorders in pregnancy. Hypertension. 2009;53:812-8.

39. Poon LC, Akolekar R, Lachmann R, Beta J, Nicolaides $\mathrm{KH}$. Hypertensive disorders in pregnancy: screening by biophysical and 
biochemical markers at 11-13 weeks. Ultrasound Obstet Gynecol. 2010;35:662-70.

40. Akolekar R, Syngelaki A, Sarquis R, Zvanca M, Nicolaides $K H$. Prediction of early, intermediate and late pre-eclampsia from maternal factors,biophysical and biochemical markers at 11-13 weeks. Prenat Diagn. 2011;31:66-74

41. Block-Abraham DM, Turan OM, Doyle LE, Kopelman JN, Atlas $\mathrm{RO}$, Jenkins $\mathrm{CB}$, et al. First-trimester risk factors for preeclampsia development in women initiating aspirin by16 weeks of gestation. Obstet Gynecol. 2014;123:611-7.

42. American College of Obstetricians and Gynecologists, Task Force on Hypertension in Pregnancy. Hypertension in pregnancy. Report of the American College of Obstetricians and Gynecologists' Task Force on
Hypertension in Pregnancy. Obstet Gynecol. 2013;122:1122-31.

43. World Health Organization. WHO recommendations for prevention and treatment of pre-eclampsia and eclampsia. Geneva: World Health Organization; 2011.

44. Hypertension in pregnancy: the management of hypertensive disorders during pregnancy. NICE clinical guideline 107. London: National Institute for Health and Clinical Excellence (NICE); 2010.

45. Magee LA, Pels A, Helewa M, Rey E, von Dadelszen P; Canadian Hypertensive Disorders of Pregnancy Working Group. Diagnosis, evaluation, and management of the hypertensive disorders of pregnancy: executive summary. J Obstet Gynaecol Can. 2014;36:41641. 


\section{O Papel da Aspirina na Prevenção da Pré-Eclâmpsia: Estado da Arte}

Acta Med Port 2015:28:517-524

Publicado pela Acta Médica Portuguesa, a Revista Científica da Ordem dos Médicos

Av. Almirante Gago Coutinho, 151

1749-084 Lisboa, Portugal.

Tel: +351218428215

E-mail: submissao@actamedicaportuguesa.com

www.actamedicaportuguesa.com

ISSN:0870-399X | e-ISSN: 1646-0758

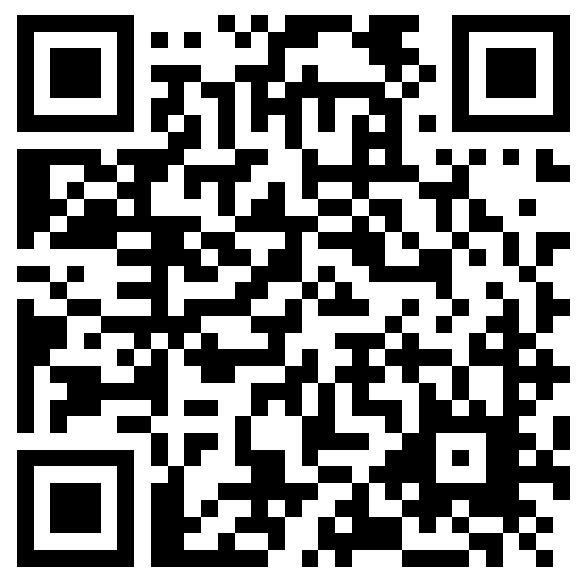

